\title{
Efeito do tratamento de superfície na resistência de união entre porcelana e uma cerâmica à base de zircônia
}

\author{
(Effect of surface treatment in the bond strength between \\ porcelain and a zirconia-based ceramic)
}

\author{
E. Lodi, F. Marks, M. Borba \\ Programa Pós-Graduação em Odontologia, Faculdade de Odontologia da Universidade de Passo Fundo, \\ BR286, Passo Fundo, RS, Brasil 99052-900 \\ marciaborba@upf.br,marcia_borb@hotmail.com
}

\begin{abstract}
Resumo
A substituição do metal utilizado como infraestrutura de restaurações protéticas pela cerâmica policristalina à base de zircônia tetragonal estabilizada por ítria (Y-TZP) resultou em dúvidas sobre a qualidade de união entre as cerâmicas de infraestrutura e de recobrimento. Assim, surgiram alguns tratamentos de superfície com o intuito de melhorar a união porcelana-Y-TZP. O objetivo deste estudo foi avaliar o efeito de um tratamento de superfície na resistência de união entre a porcelana e a Y-TZP, bem como comparar os resultados com um grupo controle metal-cerâmica. Três grupos experimentais foram avaliados $(n=10)$ : YC - infraestrutura de Y-TZP recoberta com porcelana VM9; YB - infraestrutura de Y-TZP recoberta com um agente de união e porcelana VM9; MC infraestrutura de níquel-cromo recoberta com opaco e porcelana VM13. Os corpos de prova foram confeccionados usando lâminas do material de infraestrutura, e sobre elas foi construído um cilindro de porcelana ( $3 \mathrm{~mm}$ de diâmetro, $6 \mathrm{~mm}$ de altura), sinterizado de acordo com as instruções do fabricante. Os grupos foram submetidos ao teste de cisalhamento em uma máquina de ensaio universal com velocidade de $0,5 \mathrm{~mm} / \mathrm{min}$. Após os testes, as falhas foram classificadas como adesiva, mista ou coesiva. Os dados de resistência de união $\left(\sigma_{\mathrm{f}}\right)$ foram analisados estatisticamente com Anova de um fator $(\alpha=0,05)$. As médias (desvio-padrão) de $\sigma_{\mathrm{f}}(\mathrm{MPa})$ foram: YC - 19,7 (7,8); YB - 22,5 (6,4); MC - 24,2 (6,6). Não foi encontrada diferença estatística entre os grupos (p=0,377). Houve relação entre o grupo experimental e o modo de falha. Apenas os grupos YC e YB apresentaram falha coesiva na porcelana. Concluiu-se que a infraestrutura à base de Y-TZP tem resistência de união à porcelana semelhante ao "padrão ouro" metal-cerâmica. A aplicação de um agente de união não teve influência na resistência de união entre porcelana e Y-TZP.

Palavras-chave: cerâmica, prótese parcial fixa, resistência ao cisalhamento.
\end{abstract}

\begin{abstract}
The replacement of the metal used as infrastructure in prosthetic restorations with yttria-stabilized tetragonal zirconia polycrystal $(Y-T Z P)$ raised questions regarding the quality of the bond strength between the ceramic infrastructure and veneer. Therefore, surface treatments were developed in order to improve porcelain-Y-TZP bond strength. The objective of this study was to evaluate the effect of a surface treatment on the bond strength between the porcelain veneer and Y-TZP, as well as to compare the results with a metalceramic control group. Three experimental groups were evaluated $(n=10): Y C$ - Y-TZP infrastructure veneered with VM9 porcelain; YB - Y-TZP infrastructure veneered with a bond agent and VM9 porcelain; MC - nickel-chrome infrastructure veneered with opaque and VM13 porcelain. Specimens were produced using slices of the infrastructure materials. A porcelain cylinder (3 mm diameter, $6 \mathrm{~mm}$ height) was built over the infrastructures and sintered according to the manufacturer recommendation. Specimens were subjected to a shear bond strength test in a universal testing machine at $0.5 \mathrm{~mm} / \mathrm{min}$ cross-head speed. After testing, failure modes were classified as adhesive, cohesive or combined. Shear bond strength $\left(\sigma_{f}\right)$ data were analyzed with one-way ANOVA $(\alpha=0.05) . \sigma_{f}$ mean values (standard deviation) in MPa were: YC - 19.7 (7.8); YB - 22.5 (6.4); MC - 24.2 (6.6). There was no statistical difference between groups $(p=0.377)$. There was an association among the experimental group and the failure mode. Only YC and YB groups showed porcelain cohesive failure. It was concluded that Y-TZP infrastructure show similar shear bond strength to the porcelain than the "gold standard" metal-ceramic. The application of a bond agent had no influence in the bond strength results.
\end{abstract}

Keywords: ceramic, partial fixed denture, shear strength.

\section{INTRODUÇÃO}

Embora as próteses fixas metalocerâmicas sejam consideradas o "padrão ouro", as próteses totalmente cerâmicas vêm ocupando cada vez mais espaço no mercado devidoà introduçãona Odontologia da tecnologia CAD-CAM (computer aided design - computer aided manufacturing) e de cerâmicas policristalinas com alta tenacidade à fratura $[1$, 2]. As próteses totalmente cerâmicas têm vantagens como a possibilidade de posicionar o término cervical do preparo na mesma altura da margem gengival livre, levando a um melhor resultado estético e evitando a invasão do espaço 
biológico. Além disso, suas propriedades ópticas favorecem uma aparência mais natural da restauração, tem baixa condutividade térmica e são altamente biocompatíveis [1, 3,4]. Dentre as cerâmicas policristalinas disponíveis para a confecção de restaurações com a tecnologia CAD-CAM, destaca-se a zircônia tetragonal estabilizada por ítria (Y-TZP). A Y-TZP apresenta comportamento mecânico superior às demais cerâmicas odontológicas devido a seu conhecido mecanismo de tenacificação por transformação de fase [57]. A Y-TZP apresenta valores médios de resistência à flexão variando entre 700 e $1500 \mathrm{MPa}$ e tenacidade à fratura entre 4,4 e 9,4 MPa.m ${ }^{1 / 2}[2,4,5,8-10]$. Essa cerâmica é indicada para a construção de infraestruturas de coroas e próteses parciais fixas (PPFs) em todas as regiões da cavidade oral. Entretanto, suas propriedades ópticas não são favoráveis. No geral, apresenta uma cor branca e opaca, sendo necessário o recobrimento com uma cerâmica vítrea, como a porcelana, para alcançar a estética do dente natural [3,4]. No entanto, um dos principais problemas clínicos observados é a fratura desta porcelana de recobrimento [1, 11-13]. Embora os lascamentos pequenos possam ser reparados sem a necessidade de remoção da prótese, os grandes (envolvendo fratura de cúspides ou contatos interproximais) levam à necessidade de troca da restauração. Uma recente revisão sistemática mostra que as taxas de fratura e lascamento da porcelana de recobrimento de coroas e PPFs à base de Y-TZP são maiores do que as taxas encontradas para as metalocerâmicas [11, 12].

Entre os fatores que podem estar associados a essas falhas são citados a falta de suporte da camada de porcelana pelo material de infraestrutura [14], baixa resistência e tenacidade à fratura da porcelana $[15,16]$, incompatibilidade térmica entre os materiais [17], protocolo de resfriamento da porcelana que pode induzir tensões residuais deletérias [18] e baixa resistência de união entre a infraestrutura e a porcelana [19]. Especificamente com relação à resistência de união entre Y-TZP e porcelana, os estudos são controversos. Algumas investigações que compararam a resistência de união ao cisalhamento da porcelana com a infraestrutura de Y-TZP (sem tratamento de superfície) e com a infraestrutura de metal concluíram que o grupo com infraestrutura de metal apresenta resistência de união superior à porcelana [19-22]. Por outro lado, um estudo encontrou resistência de união igual quando Y-TZP ou metal foram utilizados [23]. Entretanto, conclusões a respeito da superioridade da união metal-cerâmica são limitadas por variações metodológicas dos estudos, envolvendo diferentes tipos de ligas, variados tratamentos de superfície e porcelanas de diferentes composições.

Com o objetivo de melhorar essa resistência de união entre Y-TZP e porcelana foram propostos tratamentos de superfície como jateamento com partículas de alumina, recobrimento com sílica (silicatização), abrasão com brocas, aplicação de agente de união ou liner de porcelana, tratamento experimental com pó de zircônia, irradiação com laser e combinações desses tratamentos. Os estudos observaram que o comportamento encontrado foi dependente do tipo de tratamento e tipo de cerâmica, porém, nem sempre havia uma melhora na união entre os materiais [21,24-28]. Além disso, o uso de tratamentos que geram tensões na superfície da Y-TZP pode ser contraindicado devido à possível indução de transformação da fase tetragonal para monoclínica, que leva à degradação das suas propriedades mecânicas em baixas temperaturas (low temperature degradation - LTD) $[6,7]$. Os diferentes tratamentos de superfície buscam melhorar a união entre os dois tipos de cerâmica através de retenção micromecânica, aumento da capacidade de molhamento (diminuindo o ângulo de contato) e união química. Para os agentes de união espera-se que mecanismos químicos (ligação atômica entre moléculas da superfície da Y-TZPe do agente de união) e físicos (diminuição da tensão superficial) atuem. Assim, o objetivo do presente estudo foi avaliar a resistência de união ao cisalhamento entre a porcelana e a Y-TZP testando as seguintes hipóteses: (1) a resistência de união entre Y-TZP e porcelana é semelhante à união entre metal e porcelana; (2) não existe influência do uso de um agente de união da resistência ao cisalhamento da Y-TZP à porcelana.

\section{MATERIAIS E MÉTODOS}

Confecções dos corpos de prova: dois materiais de infraestrutura foram avaliados, uma cerâmica policristalina à base de zircônia tetragonal estabilizada com ítria (Y-TZP, Vita In-Ceram YZ, Vita Zanhfabrik, Alemanha) e uma liga metálica de níquel-cromo (Wironia ${ }^{\circledR}$ light, BEGO Bremer

Tabela I - Ciclos de sinterização da Y-TZP, das porcelanas VM9 e VM13, e do agente de união Effect Bonder. [Table I - Sintering cycles for Y-TZP, VM9 and VM13 porcelains, and Effect Bonder bond agent.]

\begin{tabular}{cccccccc}
\hline Material & $\begin{array}{c}\text { Temperatura } \\
\text { inicial } \\
\left({ }^{\circ} \mathrm{C}\right)\end{array}$ & $\begin{array}{c}\text { Tempo de } \\
\text { pré-secagem } \\
(\mathrm{min})\end{array}$ & $\begin{array}{c}\text { Tempo de } \\
\text { aquecimento } \\
(\mathrm{min})\end{array}$ & $\begin{array}{c}\text { Taxa de } \\
\text { aquecimento } \\
\left({ }^{\circ} \mathrm{C} / \mathrm{min}\right)\end{array}$ & $\begin{array}{c}\text { Temperatura } \\
\text { final } \\
\left({ }^{\circ} \mathrm{C}\right)\end{array}$ & $\begin{array}{c}\text { Manutenção } \\
\text { da temperatura } \\
\text { final }(\mathrm{min})\end{array}$ & $\begin{array}{c}\text { Tempo } \\
\text { de vácuo } \\
(\mathrm{min})\end{array}$ \\
\hline Effect Bonder & 500 & 6 & 6,0 & 80 & 980 & 1 & 6,00 \\
VM9 & 500 & 6 & 6,0 & 55 & 910 & 1 & 7,27 \\
VM13 & 500 & 6 & 6,6 & 55 & 880 & 1 & 6,55 \\
Y-TZP & 500 & - & 100 & 5 & 1000 & 15 & - \\
\hline
\end{tabular}

Fonte: Vita Zahnfabrik, manual de instruções: 09.14. 
Goldschlägerei Wilh. Herbst GmbH \& Co. KG, Bremen, Alemanha). As infraestruturas de Y-TZP foram produzidas a partir do corte de blocos de CAD-CAM em secções de $1,5 \mathrm{~mm}$ de espessura utilizando uma cortadora metalográfica (Miniton, Struers, Copenhagen, Denmark). Após, as secções foram polidas com lixas d'água de granulação 800 e 1200 . Seguiu-se a sinterização em forno específico do sistema (Zyrcomat, Vita Zahnfabrik, Alemanha), seguindo o ciclo indicado pelo fabricante (Tabela I). As dimensões finais das lâminas de Y-TZP foram de 11,0 mm x 5,5 mm x 1,2 $\mathrm{mm}$. Para as infraestruturas de metal, lâminas de cera foram esculpidas com as dimensões de $8 \mathrm{~mm}$ x $9 \mathrm{~mm}$ x 1,2 mm e incluídas em revestimento. $\mathrm{O}$ conjunto foi levado ao forno para realizar a queima da cera a $840{ }^{\circ} \mathrm{C}$. A liga metálica foi aquecida até $1350^{\circ} \mathrm{C}$ e injetada no molde de revestimento. Após a remoção das infraestruturas do anel de fundição, as mesmas foram submetidas a um ciclo térmico para produzir a camada de óxidos necessária para a união química com a porcelana. Após, as infraestruturas foram divididas em três grupos experimentais $(\mathrm{n}=10)$ : YC - infraestrutura de Y-TZP recoberta com porcelana VM9 (Vita VM9, Vita Zanhfabrik, Alemanha); YB - infraestrutura de Y-TZP recoberta com uma camada de agente de união (Effect Bonder, Vita Zanhfabrik, Alemanha) e porcelana VM9; e MC - infraestrutura de $\mathrm{NiCr}$ recoberta com porcelana VM13 (Vita VM13, Vita Zanhfabrik, Alemanha).

Para produzir os corpos de prova para o teste de cisalhamento foi utilizada uma matriz bipartida $(6 \mathrm{~mm}$ de diâmetro e $3 \mathrm{~mm}$ de altura), que foi posicionada sobre as infraestruturas. O pó de porcelana foi misturado com água destilada e condensado dentro da matriz. $\mathrm{O}$ excesso de água foi removido com papel absorvente. A matriz foi removida deixando um cilindro de porcelana sobre as infraestruturas. Este conjunto foi levado ao forno (Vita Vacumat 40, Vita Zahnfabrik, Alemanha) para sinterização da porcelana de acordo com as instruções do fabricante (Tabela I). Finalizado o ciclo de sinterização, o forno foi aberto e a cerâmica foi resfriada à temperatura ambiente. $\mathrm{O}$ grupo YC recebeu apenas o recobrimento com uma camada da porcelana VM9. Já no grupo YB, antes da aplicação da porcelana VM9, foi aplicado o agente de união Effect Bonder (Vita Zanhfabrik, Alemanha) e sinterizado de acordo com o ciclo do fabricante (Tabela I). Para o grupo MC, foi aplicada uma camada de opaco (Vita VM13 Wash Opaque, Vita Zahnfabrik, Alemanha) e sinterizada previamente à aplicação da porcelana. Após a sinterização da porcelana sobre as infraestruturas, os corpos de prova foram incluídos em cilindros de PVC ( $10 \mathrm{~mm}$ de altura e $20 \mathrm{~mm}$ de diâmetro) com resina acrílica autopolimerizável JET (Artigos Odontológicos Clássico, São Paulo, SP) para possibilitar o posicionamento dos corpos de prova na máquina de ensaios universal.

Teste de resistência de união e análise do modo de falha: foi realizado o teste de cisalhamento para avaliar a resistência de união entre a porcelana e o material de infraestrutura, utilizando o dispositivo Bencor Multi-T, em uma máquina de ensaio universal (EMIC, DL 2000, São José dos Pinhais, PR, Brasil) com velocidade de $0,5 \mathrm{~mm} / \mathrm{min}$. A carga de fratura $(\mathrm{F}$, em $\mathrm{N})$ foi registrada e a área de união mensurada (A, em $\mathrm{mm}^{2}$ ). A resistência ao cisalhamento $\left(\sigma_{\mathrm{f}}\right.$, em MPa) foi calculada com a fórmula: $\sigma_{\mathrm{f}}=\mathrm{F} / \mathrm{A}$. Após os ensaios, a superfície de fratura dos corpos de prova foi analisada em estereomicroscópio e a falha foi classificada como: adesiva, quando não permanecia nenhum material na superfície da infraestrutura; mista, quando a superfície da infraestrutura estava exposta, mas permaneciam alguns resíduos de porcelana; coesiva, quando a superfície do material de infraestrutura estava completamente recoberta por porcelana. Os dados de resistência de união foram analisados estatisticamente com teste Anova de um fator $(\alpha=0,05)$. O teste Qui-quadrado foi realizado para verificar a relação entre os grupos experimentais e o modo de falha $(\alpha=0,05)$.

\section{RESULTADOS}

Os resultados de resistência de união ao cisalhamento $\left(\sigma_{\mathrm{f}}\right)$ para os grupos experimentais podem ser observados na Fig. 1. Não foi encontrada diferença estatística entre os grupos $(\mathrm{p}=0,377)$. Na Tabela II encontra-se a frequência de cada modo de falha para cada grupo experimental. Foi encontrada

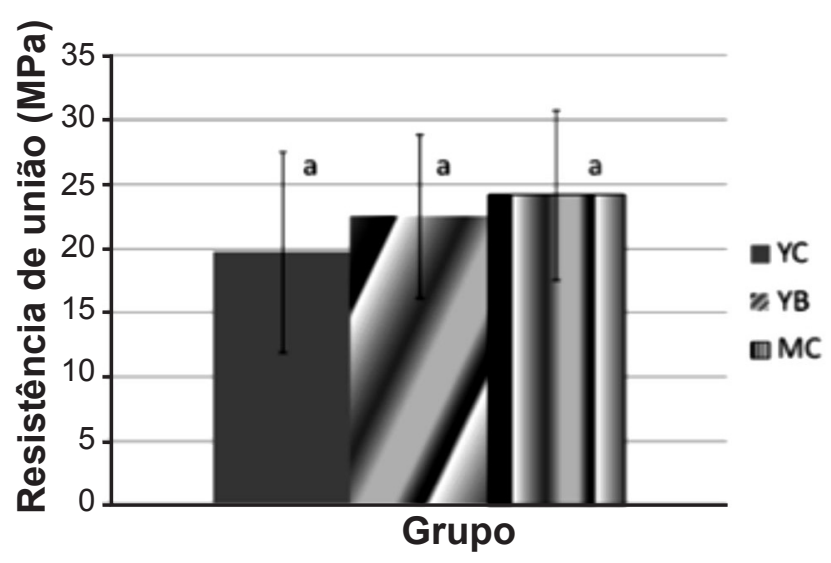

Figura 1: Resultados de resistência de união ao cisalhamento para os grupos experimentais. Letras iguais indicam que não há diferença estatística $(\mathrm{p} \geq 0,05)$.

[Figure 1: Shear bond strength data for the experimental groups. Similar letters indicate that there is no statistical difference $(p \geq 0.05)$.]

Tabela II - Frequência do modo de falha para cada grupo experimental.

[Table II - Frequency of failure mode for each experimental group.]

\begin{tabular}{cccc}
\hline Grupo & Adesiva & Mista & Coesiva em porcelana \\
\hline MC & $3(30 \%)$ & $7(70 \%)$ & $0(0 \%)$ \\
YC & $0(0 \%)$ & $7(70 \%)$ & $3(30 \%)$ \\
YB & $0(0 \%)$ & $5(50 \%)$ & $5(50 \%)$ \\
\hline
\end{tabular}




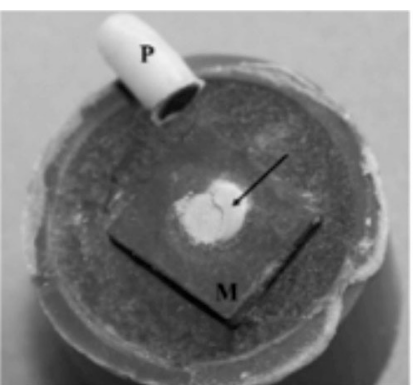

a)

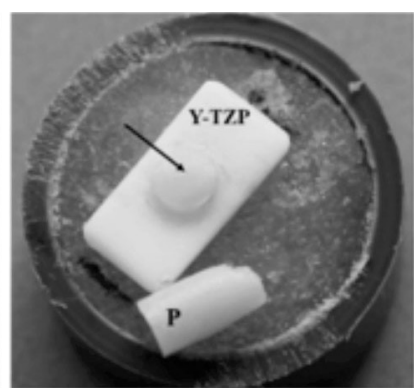

b)
Figura 2: Imagens representativas dos modos de falha mais frequentes: (a) falha do tipo mista de um corpo de prova do grupo MC (a flecha aponta para a porcelana remanescente na interface de união); (b) falha do tipo coesiva em porcelana de um corpo de prova do grupo YC (a flecha aponta para a porcelana recobrindo completamente a interface de união). [Figure 2: Representative images of the most frequent failures modes: (a) combined failure of a specimen from $M C$ group (the arrow point to the residual porcelain at the interface); (b) cohesive porcelain failure of a specimen from YC group (the arrow point to the porcelain completely covering the interface).]

relação entre o grupo experimental e o modo de falha $(\mathrm{p}=0,025)$. Apenas o grupo MC apresentou falha adesiva. Os grupos com infraestrutura de Y-TZP apresentaram falha mista e coesiva em porcelana. Os modos mais frequentes de falha podem ser observados na Fig. 2.

\section{DISCUSSÃO}

A falha clínica da cerâmica de recobrimento de restaurações à base de zircônia é motivo de investigações e leva à procura por novas estratégias para melhorar a união entre essas duas cerâmicas $[11,12,20]$. No presente estudo, não houve diferença nos valores de resistência de união obtidos pelos grupos com infraestrutura de Y-TZP e pelo grupo metalocerâmico, aceitando a primeira hipótese. Esse resultado divergiu de parte da literatura, que relata maior resistência de união para o grupo metalocerâmico, especialmente quando a infraestrutura de Y-TZP não foi submetida a nenhum tratamento de superfície [19-22]. Vários fatores podem influenciar a resistência de união entre metal e cerâmica, como o tempo e a temperatura de queima, a textura de superfície do metal (microrretenção), compatibilidade entre os coeficientes de expansão térmico linear (CET), uso de agentes de união e qualidade da camada de óxidos $[17,22]$. Assim, é difícil comparar os resultados de diferentes estudos. Três estudos que relataram maior resistência de união para o conjunto metal-cerâmica utilizaram ligas à base de CrCo e AuPtPd [19,20]. De acordo com a literatura existem diferenças de resistência de união também entre diferentes ligas metálicas, sendo que as ligas nobres apresentam maiores valores [22]. Cabe ressaltar que, nesta pesquisa, foram realizados os protocolos indicados pelos fabricantes da liga metálica de $\mathrm{NiCr}$ e da porcelana, sendo realizadas as etapas de oxidação do metal e aplicação da camada de opaco previamente ao recobrimento com a porcelana. Com relação ao modo de falha, $70 \%$ foi mista, o que sugeriu uma boa união do metal com a porcelana. A presença de algumas falhas do tipo adesiva pode estar relacionada a diferenças nas propriedades mecânicas dos dois materiais.

A adesão entre o metal e a porcelana é atribuída principalmente à retenção micromecânica e à união química resultante da oxidação do metal e da interdifusão de íons. Já os mecanismos de união da Y-TZP à porcelana não estão bem explicados na literatura. As cerâmicas diferem em sua composição química, microestrutura e propriedades, como o módulo de elasticidade e o CET. A compatibilidade entre diferentes cerâmicas é promovida pela habilidade da porcelana de molhar e escoar nas irregularidades de superfície da infraestrutura. A união entre cerâmicas seria um efeito de interações físico-químicas que ocorrem na interface; sugere-se pouca contribuição do mecanismo de retenção micromecânica quando a superfície da Y-TZP não é tratada $[17,22]$. A diferença entre o CET das cerâmicas e a taxa de resfriamento podem gerar tensões de tração ou compressão na interface. Indica-se que essa diferença entre CET seja menor ou igual a $1 \times 10^{-6}{ }^{\circ} \mathrm{C}^{-1}$ para que não ocorra fratura da porcelana ou falha de união por causa do desenvolvimento de tensões de tração radiais ou tangenciais $[17,18]$. No presente estudo, foi utilizado o material de recobrimento compatível com cada tipo de infraestrutura, sendo a diferença entre CET dentro dos limites recomendados. A baixa difusividade térmica da Y-TZP também pode levar à formação de tensões residuais na porcelana durante o resfriamento, o que poderia explicar a fina camada de porcelana presente na superfície de grande parte dos corpos de prova de Y-TZP [29].

Defeitos na interface e a contração de sinterização da porcelana também podem influenciar a resistência de união entre as cerâmicas [17]. Assim, a técnica de aplicação da porcelana e a habilidade do técnico de prótese dental afetam a qualidade da união e também podem explicar as diferenças de resultados encontrados na literatura [19]. Os estudos diferem em relação ao número de aplicações da porcelana (queima), uso de um ciclo final de glaze e protocolo de resfriamento após a sinterização [20]. No presente estudo, não foram encontrados poros ou inclusões na interface e nenhum corpo de prova dos grupos de infraestrutura de Y-TZP apresentou modo de falha adesivo, o que indicou uma boa compatibilidade entre as cerâmicas estudadas. Ainda, foi realizada apenas uma aplicação da porcelana e não foi feito o ciclo de glaze, o que pode ser uma limitação do estudo já que para uso clínico as restaurações recebem várias aplicações de porcelana e são submetidas a um glaze final.

A segunda hipótese do estudo foi aceita. Não houve influência do uso de um agente de união na resistência ao cisalhamento da Y-TZP à porcelana. A literatura relata que o uso de um liner de porcelana feldspática tem pouca ou nenhuma influência na resistência de união entre Y-TZP e porcelana, mesmo quando combinado com jateamento de 
partículas $[25,27,30]$. O agente de união utilizado (Effect Bonder) é recomendado pelo fabricante para colorir a infraestrutura de Y-TZP e melhorar sua união com a porcelana VM9. Porém, a composição do material não é fornecida e seu mecanismo de união não está bem elucidado [15]. Com base nos resultados, sugere-se que a porcelana utilizada tem bom molhamento e compatibilidade com a infraestrutura de Y-TZP, o que torna o uso de um agente de união dispensável. Apesar da similaridade nos valores de resistência de união entre os dois grupos com infraestrutura de Y-TZP, houve diferença no modo de falha. O grupo tratado com o agente de união apresentou uma maior frequência de falhas coesivas na porcelana, o que sugeriu uma melhora na qualidade da união entre os materiais. Os modos de falha encontrados foram de acordo com um estudo que relatou a presença de falha adesiva para o conjunto metal-cerâmica e falha coesiva na porcelana para os grupos de Y-TZP que utilizaram um liner previamente ao recobrimento com porcelana [20].

$\mathrm{O}$ ensaio de cisalhamento pode induzir tensões não uniformes na interface, resultando em altos valores de desvio-padrão [31]. Para evitar erros metodológicos, o dispositivo de ensaio (Bencor Multi-T) utilizado na presente pesquisa garantiu o correto alinhamento dos corpos de prova e da ponta aplicadora da carga, posicionada na interface entre os materiais. Os modos de falha e os valores de resistência de união encontrados foram semelhantes aos reportados na literatura, indicando que o método foi capaz de estabelecer um ranqueamento confiável entre os grupos experimentais. Ainda, o teste de cisalhamento foi escolhido por causa da dificuldade em confeccionar corpos de prova de Y-TZP-porcelana para o ensaio de microtração, que é o método mais indicado para avaliar a resistência de união entre materiais (defeitos de interface poderiam ser introduzidos durante o corte dos corpos de prova) [22]. No ambiente oral, as restaurações estão submetidas à fadiga pela presença de cargas cíclicas, variações de temperatura e $\mathrm{pH}$, e pela umidade do meio. Por isso, esses fatores também devem ser considerados nas pesquisas. Estudos concluíram que a ciclagem térmica e mecânica e as diferentes técnicas de recobrimento (estratificada e prensada) não influenciaram na resistência de união Y-TZP e porcelana $[19,20]$.

\section{CONCLUSÕES}

As infraestruturas cerâmicas à base de Y-TZP tiveram resistência de união à porcelana semelhante às infraestruturas metálicas. O uso de um agente de união não teve influência na resistência de união entre Y-TZP e porcelana.

\section{AGRADECIMENTOS}

Os autores agradecem a colaboração do Laboratório de Prótese Dentária Coral (Passo Fundo, RS) que contribuiu para a execução dos corpos de prova desta pesquisa.

\section{REFERÊNCIAS}

[1] A. Della Bona, J.R. Kelly, J. Am. Dent. Assoc. 139 Suppl. (2008) 8S.

[2] M. Borba, M.D. Araujo, K.A. Fukushima, H.N. Yoshimura, P.F. Cesar, J.A. Griggs, A. Della Bona, Dent. Mater. 27, 7 (2011) 710.

[3] M.J. Heffernan, S.A. Aquilino, A.M. Diaz-Arnold, D.R. Haselton, C.M. Stanford, M.A. Vargas, J. Prosthet. Dent. 88 (2002) 10.

[4] Y.D. Belo, Q.N. Sonza, M. Borba, A. Della Bona, Cerâmica 59 (2013) 633.

[5] I. Denry, J.R. Kelly, Dent. Mater. 24 (2008) 299.

[6] J. Chevalier, L. Gremillard, J. Am. Ceram. Soc. 92 (2009) 1901.

[7] J.C.B. Mosele, M. Borba, Cerâmica 60, 354 (2014) 179. [8] M. Guazzato, M. Albakry, S.P. Ringer, M.V. Swain, Dent. Mater. 20 (2004) 449.

[9] J. Tinschert, D. Zwez, R. Marx, K.J. Anusavice, J. Dent. 28 (2000) 529.

[10] B. Taskonak, J. Yan, J.J. Mecholsky Jr., A. Sertgoz, A. Kocak, Dent. Mater. 24 (2008) 1077.

[11] I. Sailer, N.A. Makarov, D.S. Thoma, M. Zwahlen, B.E. Pjetursson, Dent. Mater. 31 (2015) 603.

[12] B.E. Pjetursson, I. Sailer, N.A. Makarov, M. Zwahlen, D.S. Thoma, Dent. Mater. 31 (2015) 624.

[13] C. Sax, C.H. Hammerle, I. Sailer, Int. J. Comput. Dent. 14 (2011) 183.

[14] P.H. Corazza, S.A. Feitosa, A.L. Borges, A. Della Bona, Dent. Mater. 29 (2013) 339.

[15] M. Borba, M.D. de Araújo, E. de Lima, H.N. Yoshimura, P.F. Cesar, J.A. Griggs, A.D. Bona, Dent. Mater. 27 (2011) 1259.

[16] M. Borba, Y. Duan, J.A. Griggs, P.F. Cesar, A. Della Bona, Dent. Mater. 31 (2015) 413.

[17] P. Benetti, A. Della Bona, J.R. Kelly, Dent. Mater. 26 (2010) 743.

[18] P. Benetti, J.R. Kelly, A. Della Bona, Dent. Mater. 29 (2013) 1166.

[19] P.C. Guess, A. Kulis, S. Witkowski, M. Wolkewitz, Y. Zhang, J.R. Strub, Dent. Mater. 24 (2008) 1556.

[20] H.A. Vidotti, J.R. Pereira, E. Insaurralde, A.L. Almeida, A.L. Valle, J. Dent. 41 (2013) 307.

[21] J. Teng, H. Wang, Y. Liao, X. Liang, J. Prosthet. Dent. 107 (2012) 380.

[22] B.K. Choi, J.S. Han, J.H. Yang, J.B. Lee, S.H. Kim, J. Adv. Prosthodont. 1 (2009) 129.

[23] M.V. Lopez-Molla, M.A. Martinez-Gonzalez, J.F. Manes-Ferrer, V. Amigo-Borras, K. Bouazza-Juanes, Med. Oral Patol. Oral Cir. Bucal 15 (2010) e919.

[24] J. Fischer, B. Stawarzcyk, A. Trottmann, C.H. Hammerle, Dent. Mater. 25 (2009) 419.

[25] R. Mosharraf, M. Rismanchian, O. Savabi, A.H. Ashtiani, J. Adv. Prosthodont. 3 (2011) 221.

[26] T. Oguri, Y. Tamaki, Y. Hotta, T. Miyazaki, Dent. Mater. J. 31 (2012) 788.

[27] H.I. Yoon, I.S. Yeo, Y.J. Yi, S.H. Kim, J.B. Lee, J.S. 
Han, J. Mech, Behav. Biomed. Mater. 40 (2014) 369. [28] O. Kirmali, A. Kapdan, A. Kustarci, K. Er, J. Prosthodont. 25 (2016) 324.

[29] F. Komine, A. Saito, K. Kobayashi, M. Koizuka, H. Koizumi, H. Matsumura, J. Oral Sci. 52 (2010) 647.
[30] N. Ereifej, F.P. Rodrigues, N. Silikas, D.C. Watts, Dent. Mater. 27 (2011) 590.

[31] R.R. Braga, J.B. Meira, L.C. Boaro, T.A. Xavier, Dent. Mater. 26 (2010) e38.

(Rec. 27/04/2016, Rev. 04/08/2016, Ac. 25/10/2016) 